\title{
Wheat beer challenge
}

\section{Reinhard Meusinger $^{1}$}

Published online: 10 July 2019

(C) Springer-Verlag GmbH Germany, part of Springer Nature 2019

We would like to invite you to participate in the Analytical Challenge, a series of puzzles to entertain and challenge our readers. This special feature of "Analytical and Bioanalytical Chemistry" (ABC) has established itself as a truly unique quiz series, with a new scientific puzzle published every three months. Readers can access the complete collection of published problems with their solutions on the ABC homepage at http://www.springer.com/abc. Test your knowledge and tease your wits in diverse areas of analytical and bioanalytical chemistry by viewing this collection.

In the present challenge, wheat beer is the topic. And please note that there is a prize to be won (a Springer book of your choice up to a value of $\epsilon 100)$. Please read on...

\section{Meet the challenge}

More than 500 years ago in 1516, the Duke of Bavaria, William IV, adopted the purity law (Reinheitsgebot), perhaps the oldest food-quality regulation still in use today. According to this law, the only allowed ingredients of beer are water, hops, and barley malt. Beer contains more than $90 \%$ water, and so the mineral ions can have a significant effect on the taste. However, the distinctive taste, smell, and appearance of beer comes from the metabolic activities of plants and yeast and are covered by the fields of biochemistry and organic chemistry.

In this challenge we are looking for a compound that gives beer its distinctive flavor. The complexity of this subject is great, which is why this challenge will be limited to wheat beer. This top-fermented beer is based on the German tradition of mixing at least $50 \%$ wheat with the malted barley. Because

Reinhard Meusinger meusi@nmr.chemie.tu-darmstadt.de

1 Institute of Organic Chemistry and Biochemistry, University of Technology Darmstadt, Alarich-Weiss-Str. 4, 64287 Darmstadt, Germany of the purity requirement, wheat beer was originally brewed only north of the Danube. Since the beginning of the seventeenth century, Weissbier has also been brewed in Bavaria, whereby only the Bavarian rulers could award brewing rights. This wheat beer monopoly (also called "wheat beer privilege") was a significant source of income for the Bavarian state. Depending on the region, this beer is variably termed "German Weißbier," "white beer," "wheat," "wheat
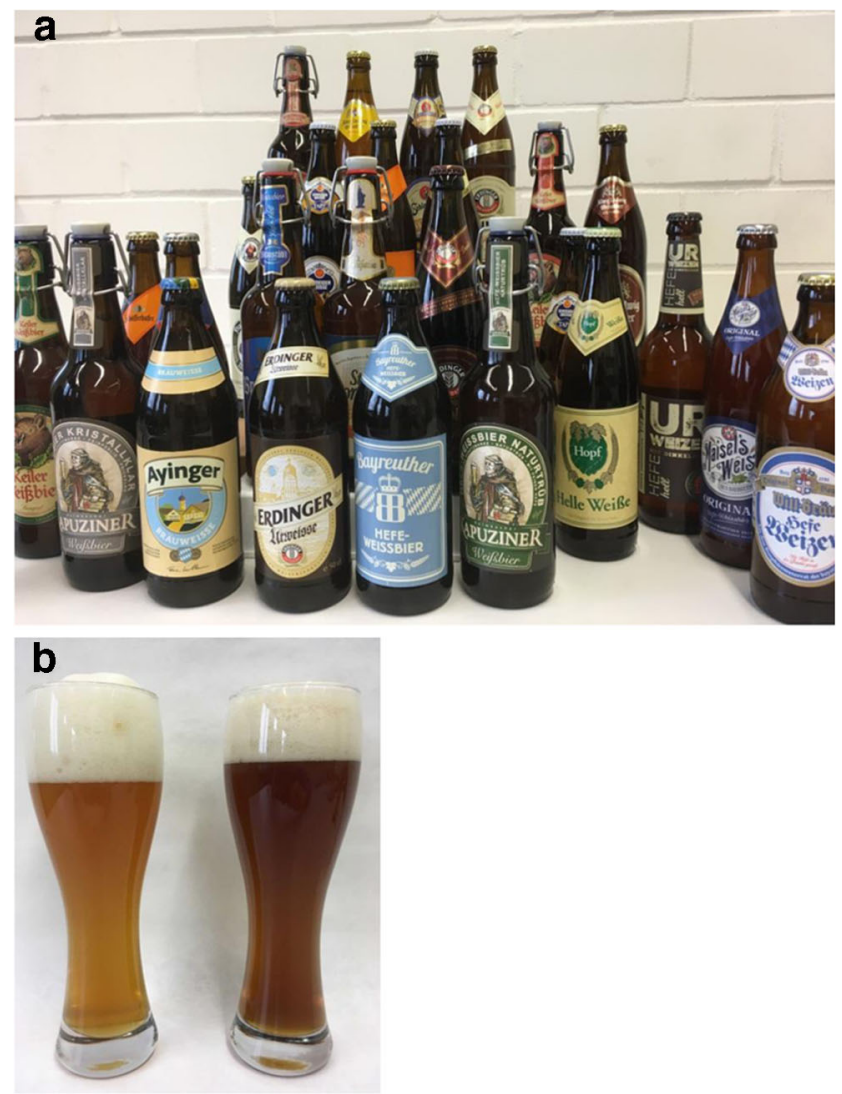

Fig. 1 a A variety of German wheat beers, different in origin, darkness, filtration, and taste. $\mathbf{b}$ Two representatives of Hefeweizen, a bright version (left) and a dark version (right), served in traditional wheat beer drinking glasses 
Fig. 2 Electron impact mass spectrum of the pure compound

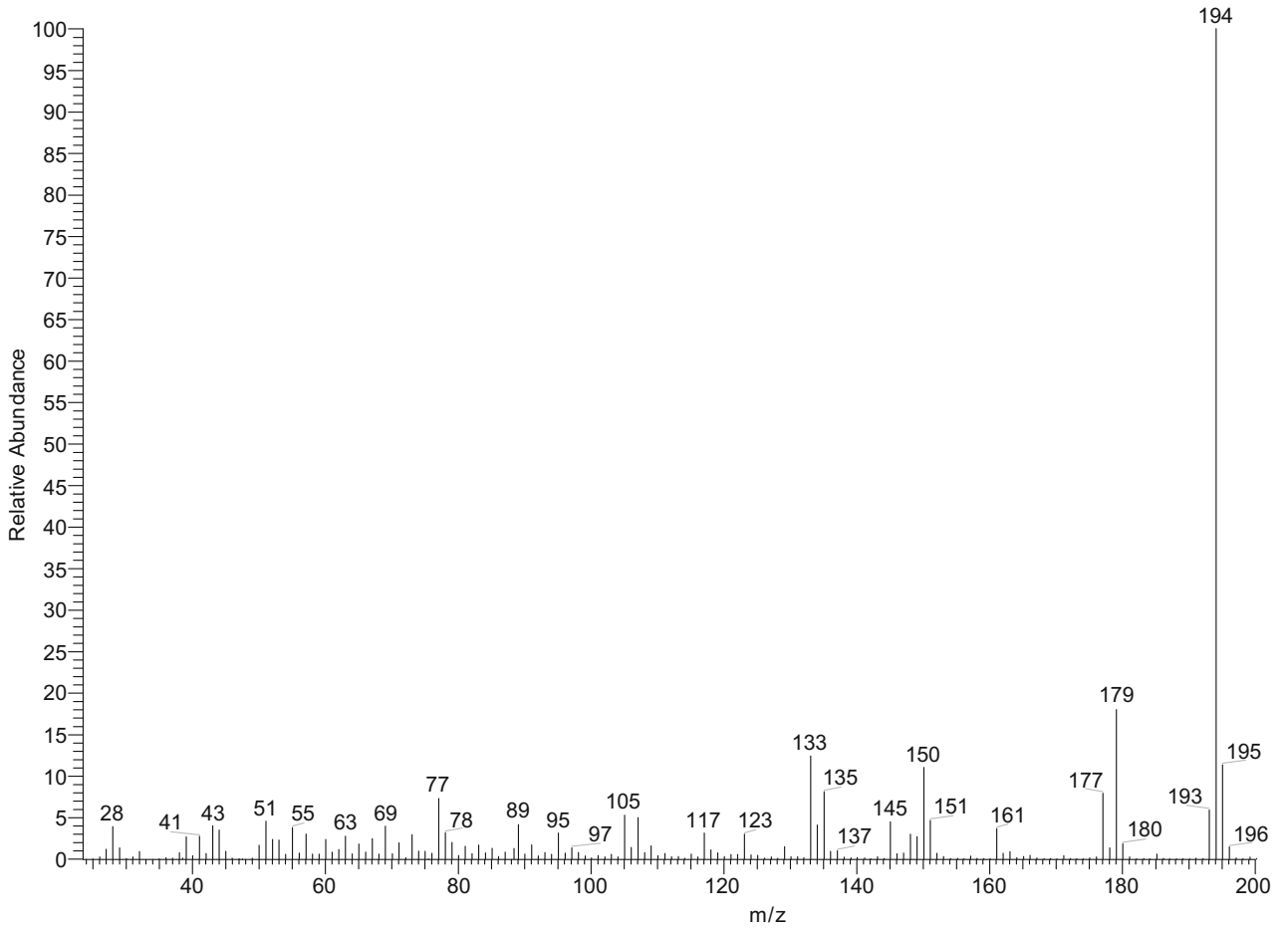

beer," or "yeast wheat." The filtered varieties are also called "crystal wheat" or "crystal white beer." Weißbier is also available in different colors, including "dark wheat" (Dunkelweizen) and "strong wheat beer" (Weizenstarkbier or Weizenbock). The dark wheat varieties are made with darker, more highly kilned wheat and barley malts (Fig. 1).

The substance we are looking for in this challenge is ubiquitous in the plant kingdom. It is found in a number of vegetable sources as a flavoring agent, and is also used by some insects for chemical signaling. Its bioavailability depends on the form in which it is present: the pure compound has limited solubility in water, and hence poor bioavailability. However, by certain strains of yeast the substance is converted to a compound that gives wheat beers their distinctive "clove" flavor.

The electron impact mass spectrum (Fig. 2), infrared spectrum (Fig. 3), and NMR spectra (Figs. 4, 5, 6, and 7) of this compound are provided for structural analysis purposes. In the mass spectrum, the strong molecular ion peak is conspicuous. The compound is very stable in a vacuum and is therefore used as a matrix for proteins in matrix-assisted laser desorption/ionization mass spectrometry analyses. All NMR spectra were obtained with $\mathrm{CDCl}_{3}$ as the solvent. As well as the ${ }^{1} \mathrm{H}$ HMR spectrum (Fig. 4) and the ${ }^{13} \mathrm{C}$ and distortionless enhancement of polarization transfer with a final ${ }^{1} \mathrm{H}$ pulse angle of $135^{\circ}$ (DEPT-135) NMR spectra (Fig. 5), four two-

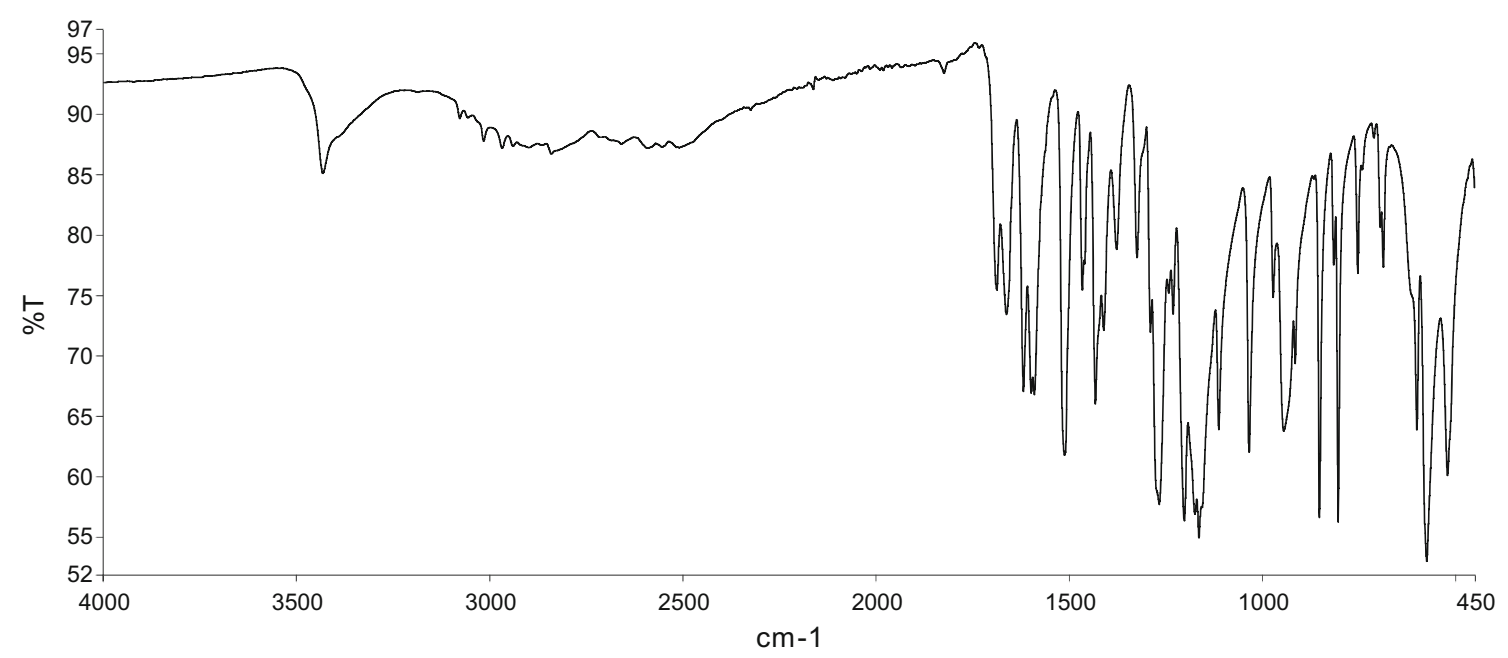

Fig. 3 Attenuated total reflection infrared spectrum of the pure compound 


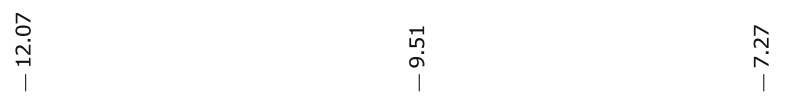
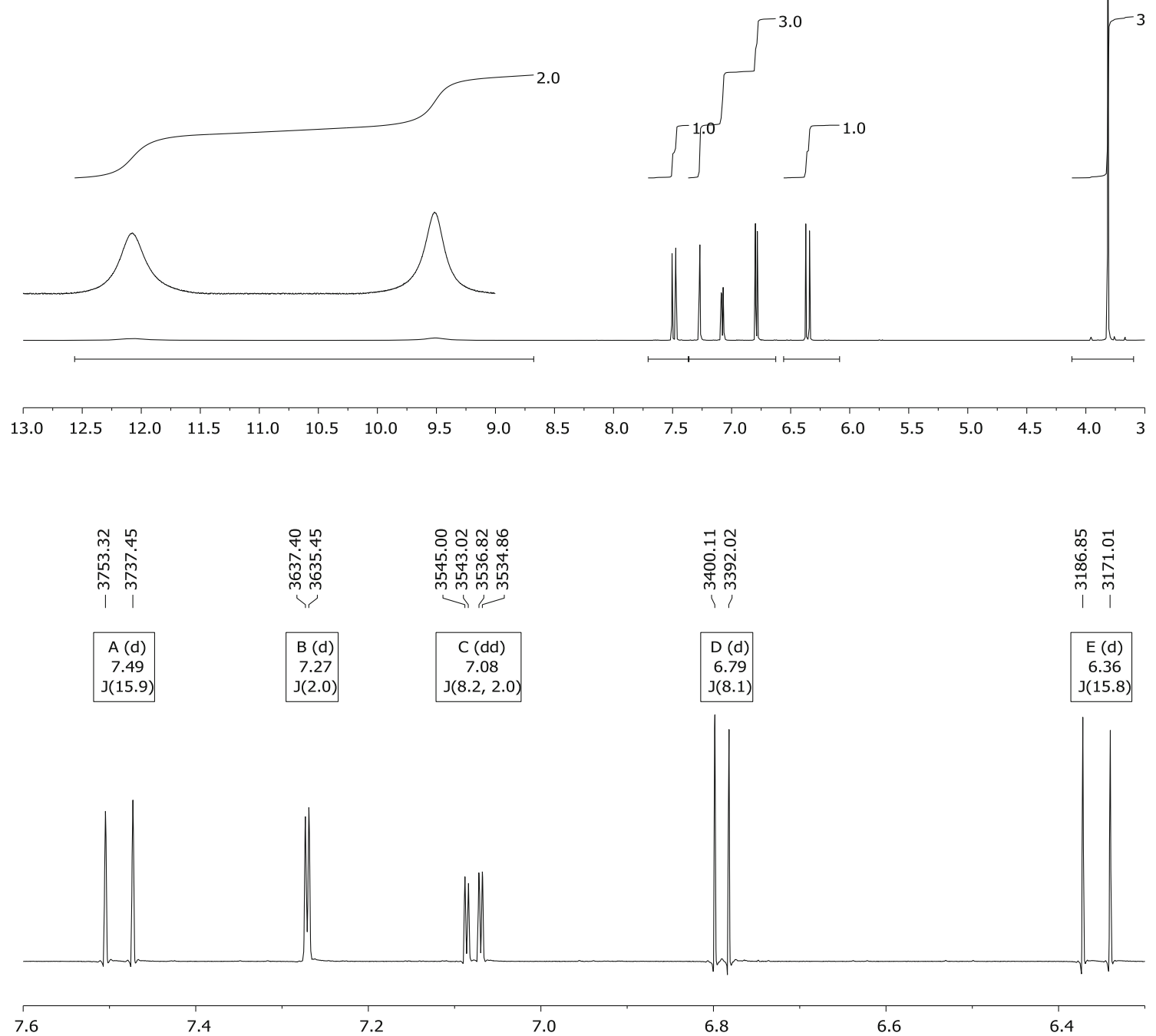

Fig. 4 The 500-MHz ${ }^{1} \mathrm{H}$ NMR spectrum of a solution in $\mathrm{CDCl}_{3}$ with respect to tetramethylsilane (top). The two broadened, low-field-shifted signals are heightened. In the spectrum at the bottom, the spreads of the multiplet signals are given after apodization of the free induction decay with exponential and Gaussian functions. Note the small splitting of $2 \mathrm{~Hz}$ caused by long-range couplings over four atomic bonds

dimensional NMR spectra are given. The scalar and dipolar $\mathrm{H}, \mathrm{H}$-correlation spectra are pictured in congruent dimensions (Fig. 6), as are the heteronuclear single-quantum correlation (HSQC) and heteronuclear multiple-bond correlation (HMBC) spectra (Fig. 7). In the HSQC spectrum, only the direct couplings $\left({ }^{1} J_{\mathrm{H}-\mathrm{C}}\right)$ are shown, whereas the couplings over two $\left({ }^{2} J_{\mathrm{H}-\mathrm{C}-\mathrm{C}}\right)$ and/or three $\left({ }^{3} J_{\mathrm{H}-\mathrm{C}-\mathrm{C}-\mathrm{C}}\right)$ covalent bonds are indicated in the more confusing HMBC spectrum. In the ${ }^{1} \mathrm{H}$ NMR spectrum, two low-field-shifted and broadened signals were detected (Fig. 4, top). The five multiplet ${ }^{1} \mathrm{H}$ NMR signals, together with their measured coupling constants, are shown in detail (Fig. 4, bottom). All two-dimensional spectra were cut in the low-field and high-field regions to allow better detection. 


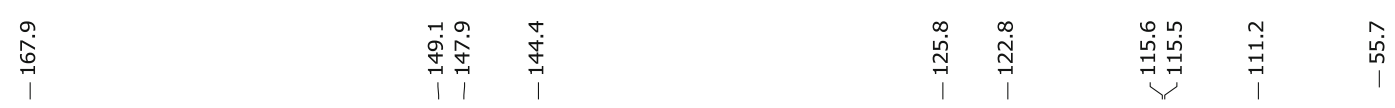

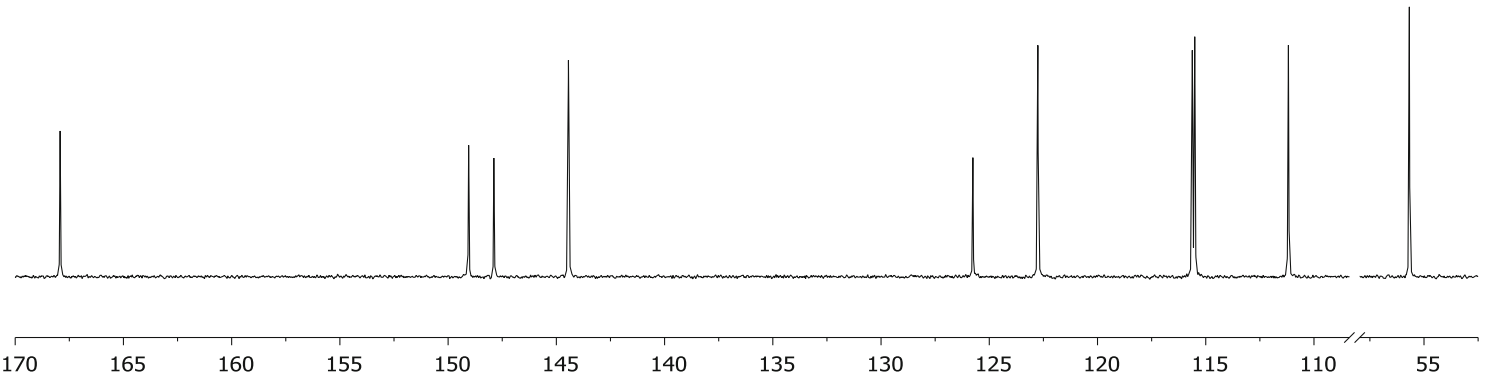

Fig. 5 The $125-\mathrm{MHz}^{13} \mathrm{C}$ NMR spectrum (bottom) and the distortionless enhancement by polarization transfer with a final ${ }^{1} \mathrm{H}$ pulse angle of $135^{\circ}$ (DEPT-135) NMR spectrum (top) with respect to $\mathrm{CDCl}_{3}$ at $77.2 \mathrm{ppm}$. Note the small shift difference of $0.1 \mathrm{ppm}$ between the two signals at $115 \mathrm{ppm}$

Fig. 6 The two-dimensional ${ }^{1} \mathrm{H}$ correlation spectroscopy (COSY) spectrum (top) and nuclear Overhauser enhancement spectroscopy (NOESY) spectrum (bottom). The truncation for the best resolution in both dimensions is marked by dashed lines

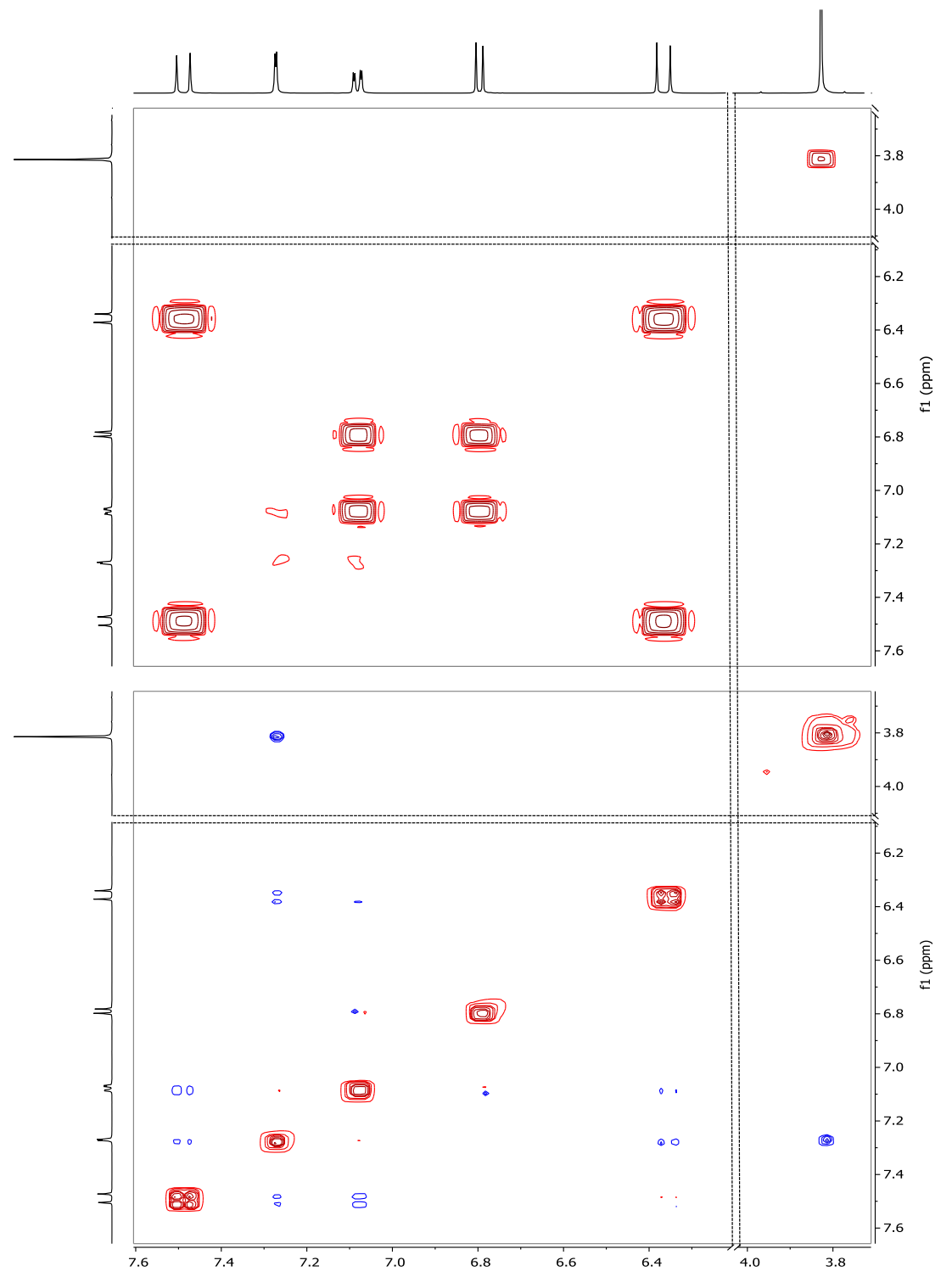


Fig. 7 The ${ }^{13} \mathrm{C}$ heteronuclear single-quantum correlation (HSQC; top) and heteronuclear multiple-bond correlation (HMBC; bottom) spectra. The truncation for the best resolution in both dimensions is marked by dashed lines

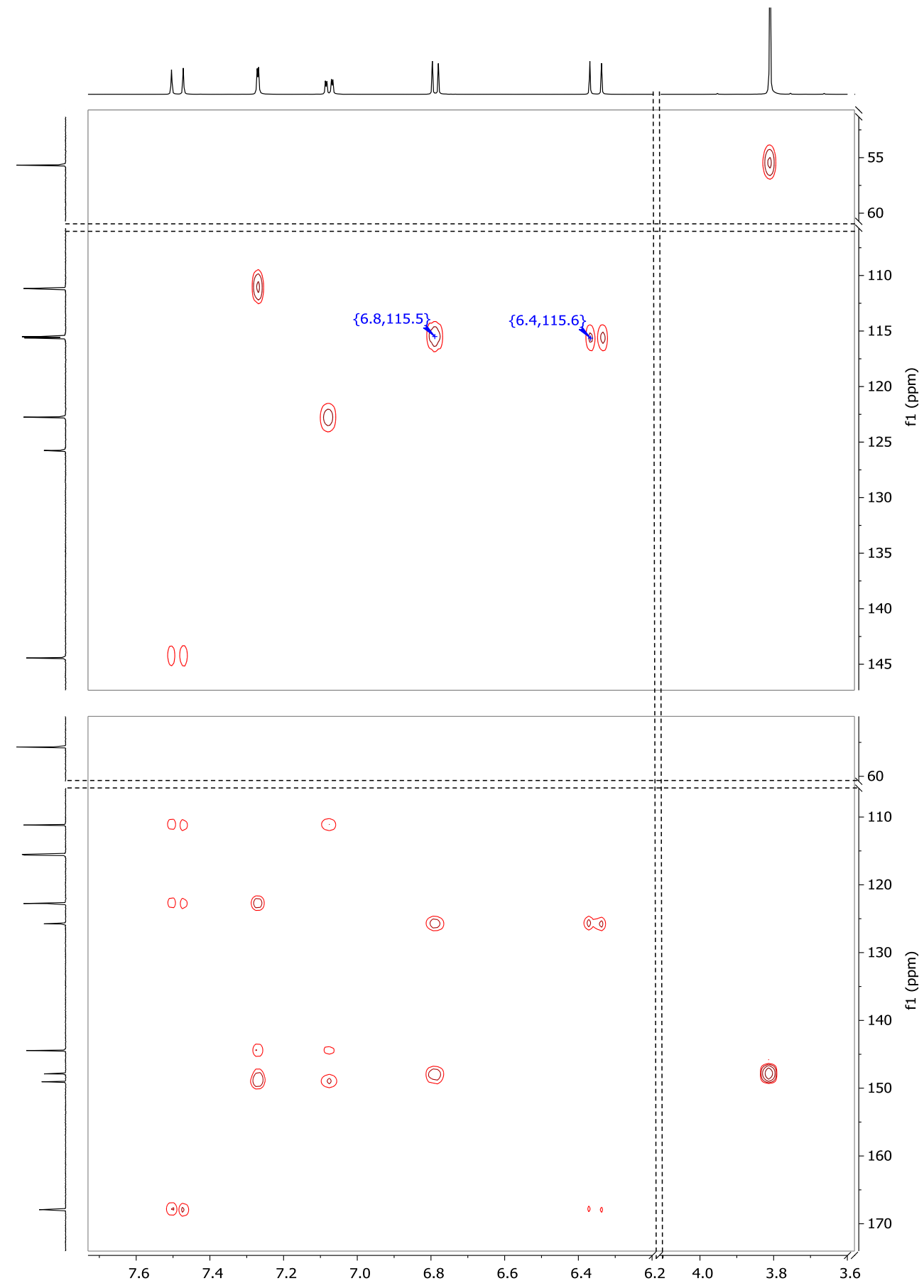

Can you identify the compound responsible for the distinctive flavor of beer?

We invite our readers to participate in the Analytical Challenge by solving the puzzle above. Please send the correct solution to abc-challenge@springer.com by October 1, 2019. Make sure you enter "Wheat beer challenge" in the subject line of your e-mail. The winner will be notified by e-mail and his/ her name will be published on the "Analytical and Bioanalytical Chemistry" (ABC) homepage at http://www. springer.com/abc and in the journal (volume 412/issue 1), where readers will find the solution and a short explanation.

The next Analytical Challenge will be published in volume 411/issue 25, October 2019. If you have enjoyed solving this Analytical Challenge, you are invited to try the previous puzzles on the $A B C$ homepage.

Publisher's note Springer Nature remains neutral with regard to jurisdictional claims in published maps and institutional affiliations. 